\title{
First Reported Case of Infective Endocarditis Caused by Community-Acquired Methicillin-Resistant Staphylococcus aureus Not Associated With Healthcare Contact in Brazil
}

\author{
Claudio Querido Fortes ${ }^{1}$, Claudia Adelino Espanha ${ }^{1}$, Flavio Pedreira Bustorff ${ }^{1}$, Bruno Cordeiro Zappa ${ }^{1}$, Adriana Lucia Pires \\ Ferreira $^{1}$, Regina Barbosa Moreira ${ }^{1}$, Nelson Gonçalves Pereira ${ }^{1}$, Vance G. Fowler Jr. $^{2}$ and Hitesh Deshmukh ${ }^{2}$ \\ ${ }^{1}$ Clementino Fraga Filho Hospital; Rio de Janeiro, RJ, Brazil; ${ }^{2}$ Duke University Medical Center; Durham, North Carolina, USA
}

\begin{abstract}
We report here the first case of endocarditis due to CA-MRSA not associated with healthcare contact in Brazil in Brazil. A previously healthy patient presented with history of endocarditis following a traumatic wound infection. Patient had multiple positive blood cultures within $72 \mathrm{~h}$ of admission and met modified Duke's criterion for infective endocarditis. The isolate was typed as Staphylococcal cassette chromosome (SCC) mec type IV and was positive for presence of PantonValentine leukocidin (PVL). Increased incidence of CA-MRSA endocarditis is a challenge for the internist to choose the best empirical therapy. Several authors have suggested an empirical therapy with both a beta-lactam and an anti-MRSA agent for serious S. aureus infections. Our patient was treated with Vancomycin and made complete recovery in 3 months.
\end{abstract}

Key-Words: Infective endocarditis, CA-MRSA, Brazil, PVL.

MRSA (methicillin-resistant Staphylococcus aureus) is a significant cause of infective endocarditis (IE) worldwide [1], and account for almost $38 \%$ of all cases of IE caused by $S$. aureus in Brazil [2]. Recently, several studies have reported MRSA infections in populations lacking previous contact with healthcare facilities. Thesecommunity-acquired MRSA(CA-MRSA) strains have been increasingly associated with skin and soft tissue infections and IE [3]. Over the past decade, MRSA has been observed sporadically as a community-acquired pathogen in Brazil [4]. We report here the first case of endocarditis due to CAMRSA in Brazil. A previously healthy patient presented with history of endocarditis following a traumatic wound infection. Patient had multiple positive blood cultures within $72 \mathrm{~h}$ of admission and met modified Duke's criterion for infective endocarditis. Following PCR for multiple $S$. aureus virulence genes and SCCmec typing, the isolate was typed as CA-MRSA.

\section{Case Report}

A 27 year old man, with a history of confusion, purpuric rashes over the extremities since 24h, history of headache, high fever, arthralgia and myalgia since 3 days was admitted to Emergency Department on October 14, 2006. One week prior to the admission, he had a traumatic wound infection on his foot which was treated with an oral cephalexin without any clinical improvement. He had no other relevant past medical history and no previous hospital admissions. He was not an intravenous drug user. Transthoracic echocardiography (TTE) and computed tomography (CT) of the head were normal. Antibiotic therapy included intravenous ceftriaxone plus vacomycin for presumed diagnosis of infective endocarditis or meningococcemia. The patient was then transferred to Hospital Universitário Clementino Fraga Filho, Rio de Janeiro for further evaluation.

Received on 19 September 2008; revised 29 October 2008.

Address for correspondence: Dr. Claudio Querido Fortes. Rua Nascimento Silva 24/102, Ipanema. Zip code: 22421-020, Rio de Janeiro, RJ, Brazil. Phone number: 5521-25220419. E-mail: c.fortes@globo.com.

The Brazilian Journal of Infectious Diseases

2008;12(6):541-543. (C) 2008 by The Brazilian Journal of Infectious Diseases and Contexto Publishing. All rights reserved.
On admission his temperature was $40.0^{\circ} \mathrm{C}$, blood pressure was 150/100 $\mathrm{mm} \mathrm{Hg}$, and pulse rate was regular at 90/min. On physical examination, conjunctival petechiae, painless hemorrhagic cutaneous lesions, palpable purpuric lesions, purulent purpura, subungual hemorrhages and digital infarct over his hands (Figures 1-3) and over his feet (Figures 4-6) were noted. Cardiovascular examination revealed a 3/6 systolic murmur at the cardiac apex and left basilar crepitations on auscultation of the chest. Abdominal examination was unremarkable except for tender hepatomegaly. Neurological examination showed nuchal rigidity but no focal neurological deficits were identified. Three sets of blood samples were collected for culture. Gram- negative diplococci were identified in the material from the purpuric lesion and antibiotic therapy with ceftriaxone and vacomycin was continued.

Two days after the admission, TTE showed vegetation on the anterior mitral valve leaflet. The patient had multiple blood cultures positive for MRSA within $72 \mathrm{~h}$ of admission and met modified Duke's criterion for infective endocarditis [5]. Since the patient did not have a history of indwelling vascular catheters, hemodialysis and exposure to a hospital or nursing home facility prior to 12 months of hospitalization the isolate was labeled as CA-MRSA. SCCmec typing was performed as described before [6] and the isolate was typed as SCCmec type IV (Figure 7). PCR was performed as described before [7] and the isolate was positive for the presence of Panton-Valentine leukocidin (PVL) [Figure 7]. The vancomycin minimal inhibitory concentration (MIC) was 2 $\mu \mathrm{g} / \mathrm{mL}$ by E-test and the isolate was susceptible to cotrimoxazole; therefore, it was added to the treatment regimen but was stopped after eleven days due to the appearance of adverse effects.

On the 15th day, emergency splenectomy was performed for the splenic abscess. After one month the patient presented with syncope. Computed tomography (CT) revealed infarction with hemorrhagic conversion and cerebral angiography revealed a mycotic aneurysm. A cerebral angiography 2 weeks later showed resolution of the mycotic aneurysm. The patient was discharged from the hospital after three months of treatment in good condition.

A PUBMED/MEDLINE search was conducted from 1966 to 2007 for english language literature with the following 
Figure 1. Digital infarct and purulent purpura on the left hand.

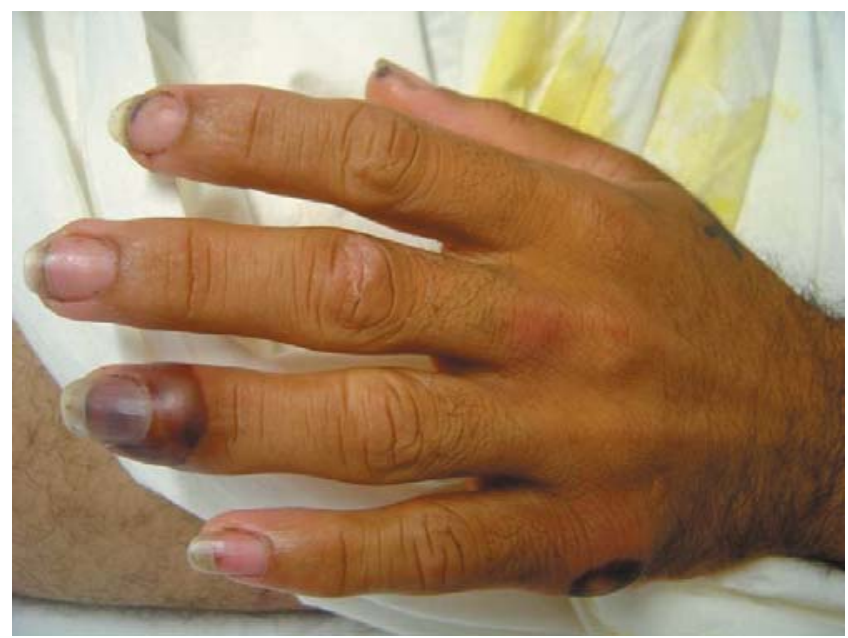

Figure 2. Hemorrhagic lesions and purulent purpura on the right hand.

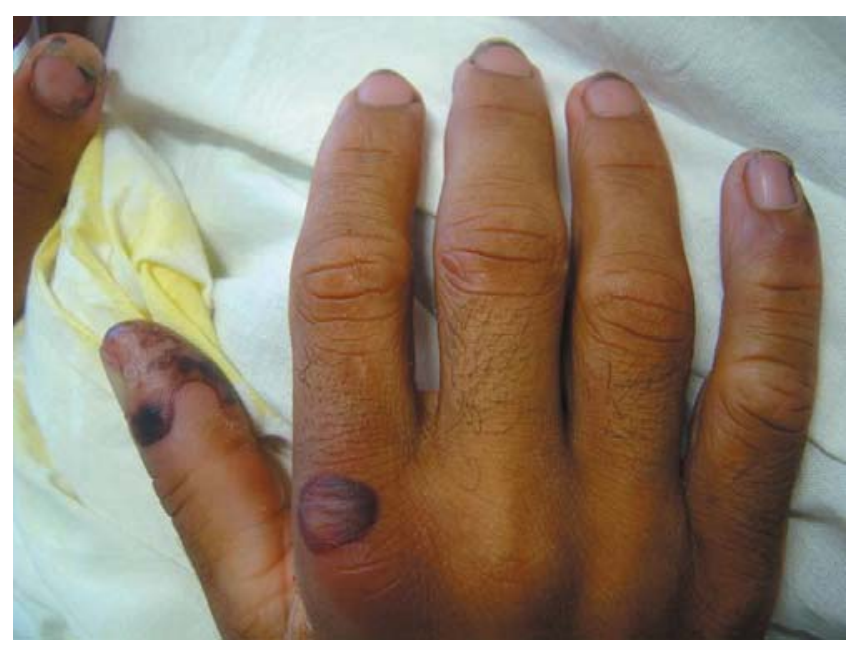

Figure 3. Hemorrhagic lesions on the right hand.

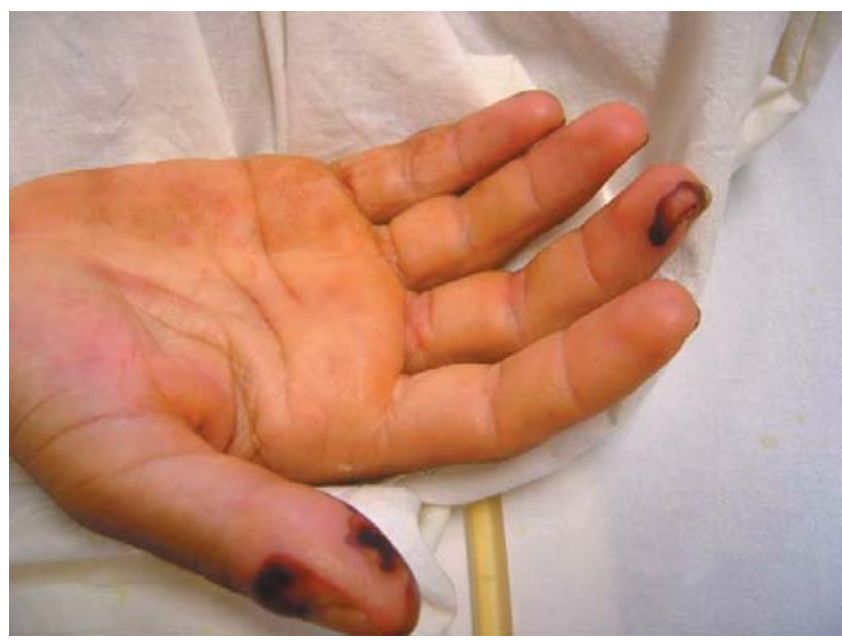

Figure 4. Hemorrhagic lesions and subungual hemorrhages on the right foot.

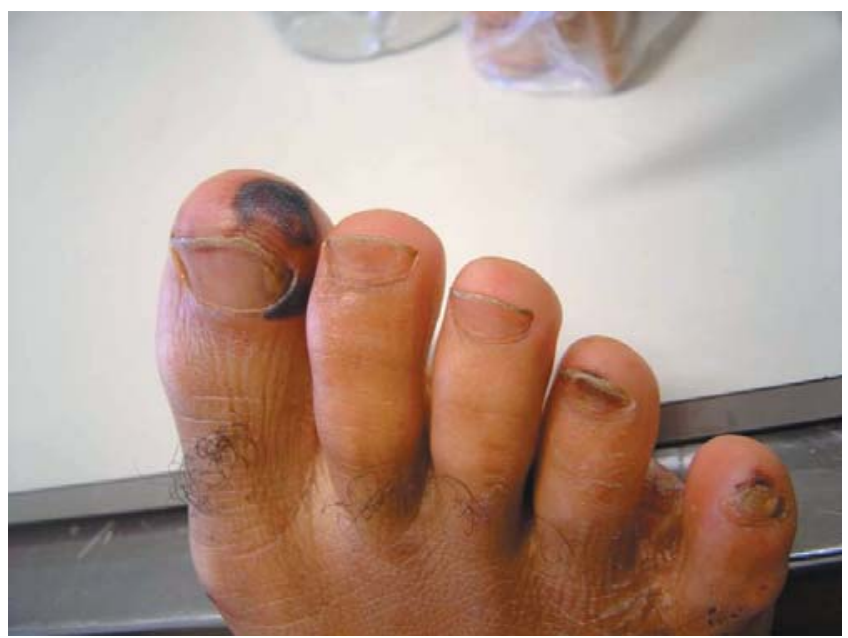

Figure 5. Hemorrhagic lesions and subungual hemorrhages on the left foot.

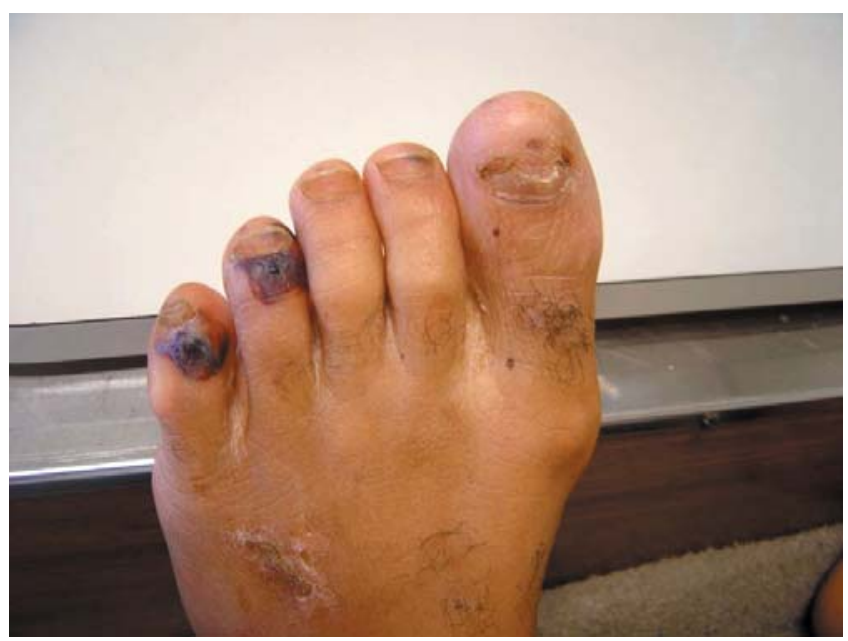

Figure 6. Purpuric rash on right foot.

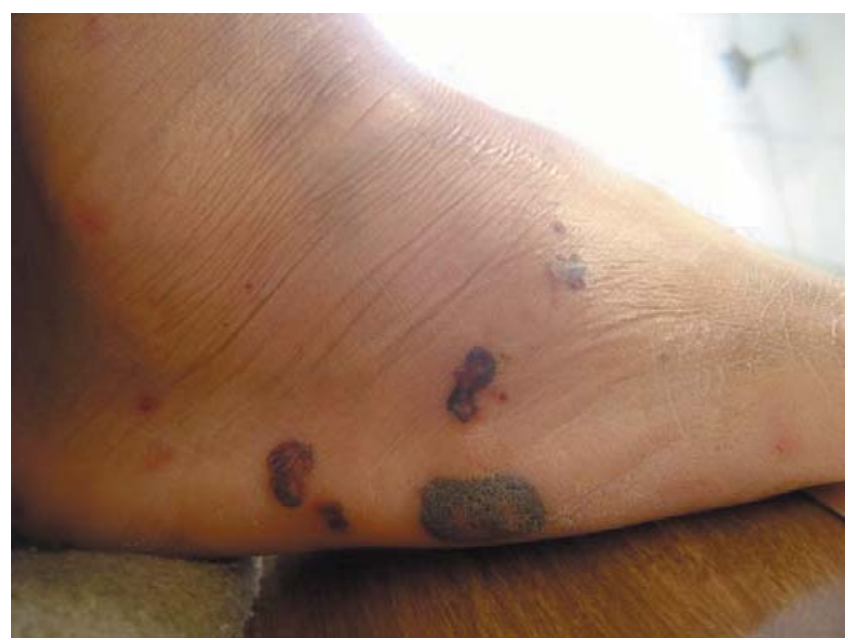


Figure 7. S. aureus strains were grown on trypticase soy agar overnight $\left(37^{\circ} \mathrm{C}\right)$, harvested by gently scraping the cells off eachplate, and resuspended in $(300 \mu \mathrm{L})$ MicroBead solution containing $20 \mu \mathrm{L}$ of lysostaphin $(1 \mathrm{mg} / \mathrm{mL})$. The genomic DNA was extracted and used in the subsequent analysis. Thirty-three bacterial determinants were examined using PCR assays; these included toxins (sea, seb, sec, sed, see, seg, seh, sei, sej, tst, eta, etb, hlg, and PVL), adhesins (bbp, clfA, clfB, cna, ebpS, fnbA, fnbB, map/eap, sdrC, sdrD, sdrE, and spa), staphylococcal cassette chromosome mec (SCCmec) types I to IV, and other virulence genes (chp, efb, icaA, V8, and arcA). A positive control for PVL was included and PCR products were analyzed by $2 \%$ agarose gel electrophoresis and photographed by a digital camera.

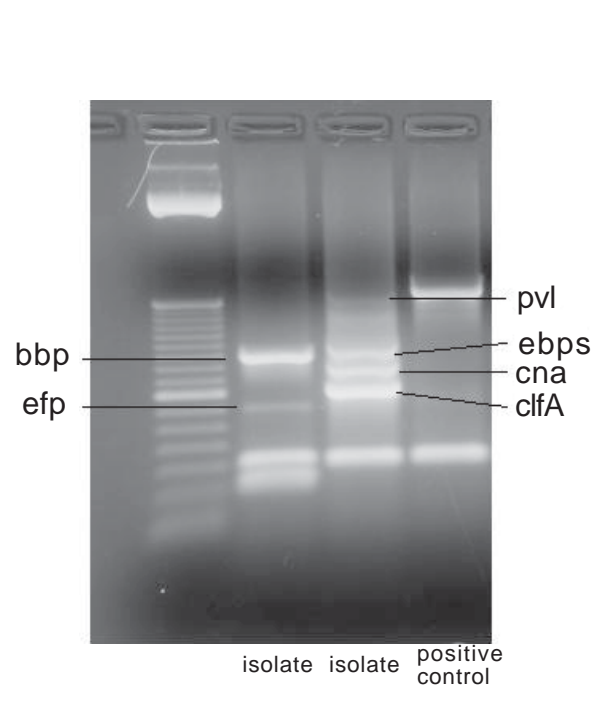

keywords and various combinations: 'community-acquired Staphylococcus aureus' and 'infective endocarditis', 'Brazil', and 'Panton-Valentine leukocidin' to identify previously reported cases of endocarditis due to CA-MRSA in Brazil.

\section{Discussion}

The incidence of infections due to CA-MRSA is rapidly increasing over the word. In Brazil, CA-MRSA was first reported by Ribeiro in 2005 [4]. Skin and soft-tissue infections, septic arthritis, epididymo-orchitis, pneumonia and surgical infection due to CA-MRSA have been reported in Brazil but to our knowledge this is the first reported case of CA-MRSA endocarditis without any kind of healthcare contact in Brazil. Cases of CA-MRSA endocarditis are likely to increase and this will pose a big challenge for the internist to choose the best empirical therapy for patients presents with acute form of endocarditis. Most of authors agree that vancomycin should be added to the empirical therapy but they also agree that vancomycin is not optimal therapy for $S$. aureus infections. Based on the available data that vancomycin is inferior to beta-lactam therapy for MSSA, Lodise Jr. et al. [8] have suggested that the empirical therapy with both a beta-lactam and an anti-MRSA agent should be considered for serious $S$. aureus infections. Conversely, Wenzel et al. [9] suggested that beta lactam antibiotic should not be used when in suspected CA-MRSA infection due to the potential risk of aggravated the infection as beta-lactam antibiotics upregulated PVL messenger RNA in several in vitro studies. So is very clear to the authors that at the present moment to choose a empirical therapy for a patient with suspected of infective endocarditis due to $S$. aureus is a extremely hard problem but while there is not more evidence that could deteriorate CA-MRSA infection, the

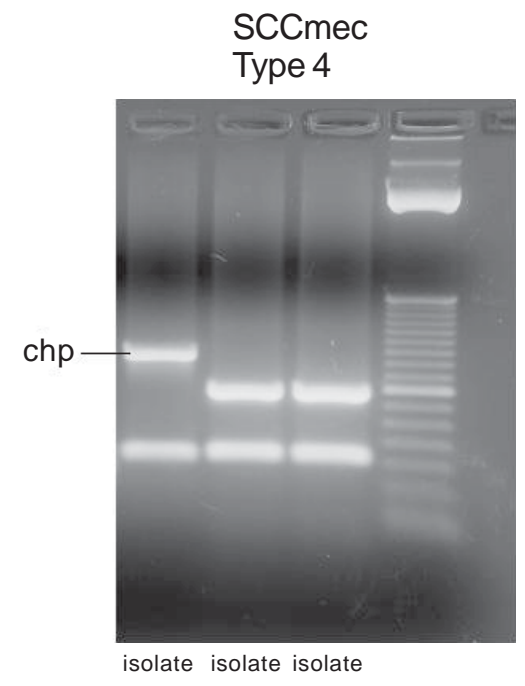

author agree with Lodise Jr. et al.that a beta-lactam antibiotic should be initiated together with an anti-MRSA agent while it is not known if it is MSSA or MRSA.

\section{Acknowledgements}

We would like to thank Dr Rosangela de Souza Rodrigues for the photographs of the patient.

\section{References}

1. Appelbaum P.C. MRSA-the tip of the iceberg. Clin Microbiol Infect 2006; Suppl 2:3-10.

2. Fowler V.G Jr., Miro J.M., Hoen B., et al. Staphylococcus aureus endocarditis: a consequence of medical progress. Jama 2005;293:3012-21.

3. Vandenesch F., Naimi T., Enright M.C., et al. Community-acquired methicillin-resistant Staphylococcus aureus carrying PantonValentine leukocidin genes: worldwide emergence. Emerg Infect Dis 2003;9:978-84.

4. Ribeiro A., Dias C., Silva-Carvalho M.C. et al. First report of infection with community-acquired methicillin-resistant Staphylococcus aureus in South America. J Clin Microbiol 2005;43:1985-8.

5. Durack D.T., Lukes A.S., Bright D.K. New criteria for diagnosis of infective endocarditis: utilization of specific echocardiographic findings. Duke Endocarditis Service. Am J Med 1994;96:200-9.

6. Vandenesch F.T., Naimi M.C., Enright G., et al. Communityacquired methicillin-resistant Staphylococcus aureus carrying Panton-Valentine leukocidin genes: worldwide emergence. Emerg Infect Dis 2003;9:978-984.

7. Peacock S.J., C.E. Moore, A. Justice, et al. Virulent combinations of adhesin and toxin genes in natural populations of Staphylococcus aureus. Infect Immun 2002;70:4987-96.

8. Lodise T.P., McKinnon P.S. Clinical and economic impact of methicillin resistance in patients with Staphylococcus aureus bacteremia. Diagn Microbiol Infect Dis 2005:52:113-22.

9. Wenzel R. P., Bearman G., Edmond M.B. Community-acquired methicillin-resistant Staphylococcus aureus (MRSA): new issues for infection control. Int J Antimicrob Agents 2007;30:210-2. 